\section{Fünf Merkmale entscheiden über die Prognose von Rückenschmerzen}

\begin{abstract}
In einem Jahr ist alles vorbei - das gilt für die Mehrheit der Patienten mit Kreuzschmerzen. Doch ein Jahr, zumal unter Schmerzen, kann lang sein. Ein Prognoseinstrument für die ersten drei Monate haben nun australische Mediziner entwickelt.
\end{abstract}

$\mathrm{R}_{\mathrm{h}}^{\mathrm{i}}$ ückenschmerzen im Lumbalbereich haben üblicherweise eine gute Prognose. Zwar konnten die in manchen Leitlinien angegebenen Erholungsraten von $90 \%$ binnen sechs Wochen in Studien nicht bestätigt werden. Dennoch darf die große Mehrheit der Patienten - etwa $70 \%$ - damit rechnen, in einem Jahr beschwerdefrei zu sein.

An jedem beliebigen Tag klagen zwischen $12 \%$ und $33 \%$ der Menschen über Kreuzschmerzen. Vor diesem Hintergrund, und weil ja auch kurzfristig etwas geschehen muss, hilft die Ein-Jahres-Prognose wenig. Ein australisches Ärzteteam um Tatiane da Silva von der Macquarie University in Sydney, hat sich deshalb damit beschäftigt herauszufinden, welche Faktoren den Verlauf von akuten Kreuzschmerzen bestimmen. Die dafür analysierten Daten stammten von 1.070 Patienten mit akuten Kreuzschmerzen. Erhoben wurden sie eine Woche, nachdem die Patienten erstmals um medizinische Hilfe nachgesucht hatten. Zu diesem Zeitpunkt waren die Probanden nicht beschwerdefrei. Ziel der Analyse war es vorherzusagen, wie es eine Woche, einen Monat und drei Monate später um die Patienten und ihre Schmerzen bestellt sein würde. Drei Monate gelten üblicherweise als Zeitspanne, ab der Rückenschmerzen als chronisch bezeichnet werden.

Fünf Merkmale, jeweils dreifach gestuft, gaben schließlich den prognostischen Ausschlag: Dauer der aktuellen Schmerzepisode (7-14, 15-23, 24-56

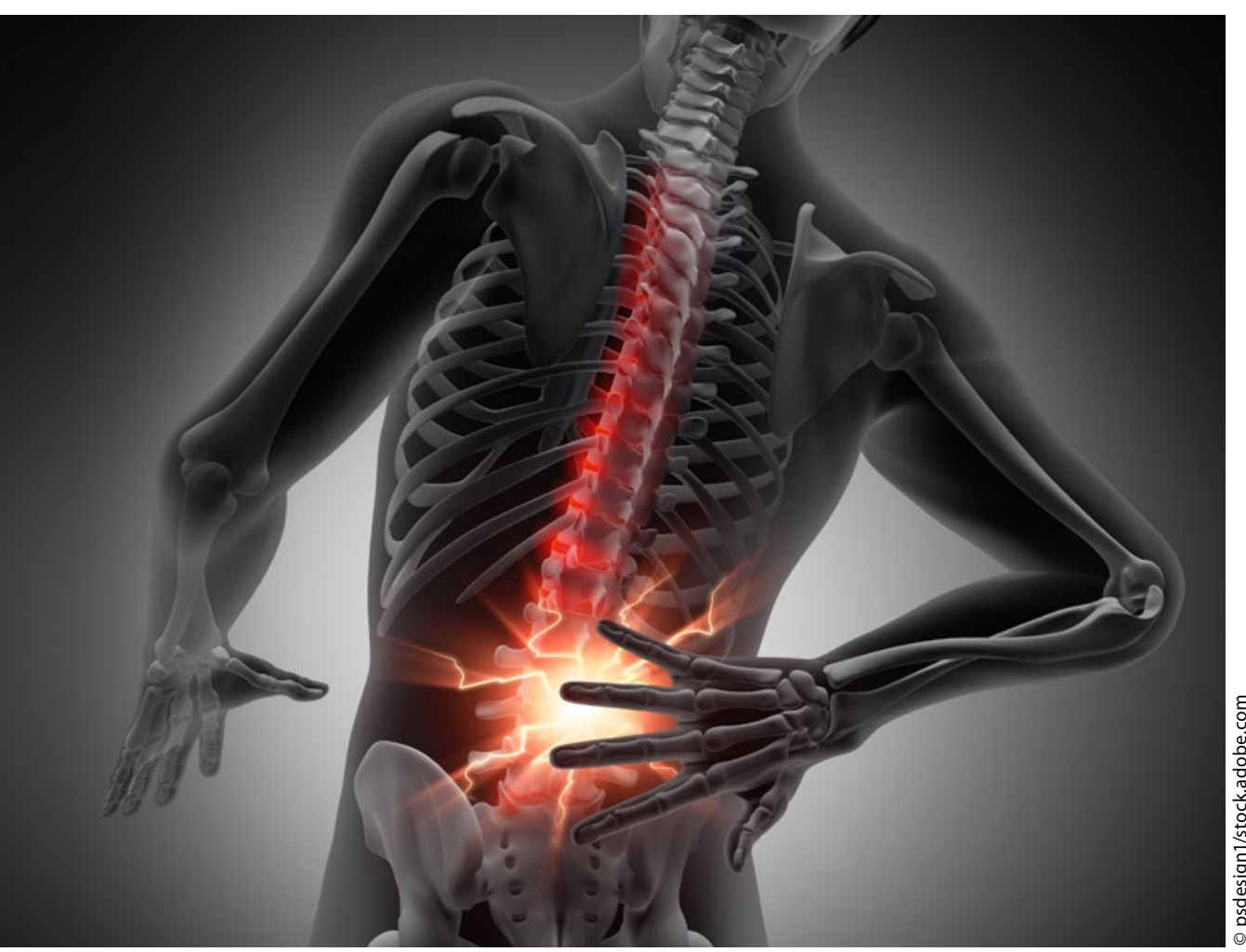

Zahl und Dauer von Rückenschmerzepisoden fließen in ein neues Prognosemodell ein.
Tage); Zahl vorangegangener Episoden $(0-2,3-8,9-150)$; Depressionsscore $(0-3$, $4-6,7-10$, je höher, desto depressiver); die Schmerzlinderung nach einer Woche (Verbesserung um vier Punkte oder mehr, um 2-3, Verbesserung um einen Punkt beziehungsweise gleichbleibend beziehungsweise Verschlechterung auf einer numerischen Rating-Skala); und die Schmerzintensität nach einer Woche (2-4, 5-7, 8-10 auf einer numerischen Rating-Skala).

Aus diesen Faktoren bildeten da Silva und ihr Team ein Vorhersagewerkzeug, das es ermöglicht, die Wahrscheinlichkeit der Gesundung eine Woche, einen Monat und drei Monate später vorherzusagen. Beispielsweise hätte ein Patienten mit einer Dauer der akuten Episode von 7-14 Tagen, 0-2 früheren Episoden, einem Depressionsscore von 0-3, mäßig bis stark gelinderten Schmerzen nach einer Woche, die sich zudem im Bereich leichter Beschwerden befinden, eine Chance von $97 \%$, dass seine Kreuzschmerzen innerhalb von drei Monaten verschwunden sind. Für einen Patienten mit langer Episodendauer, vielen vorangegangenen Kreuzwehepisoden, einem hohen Depressionsscore, keiner Linderung und starken Schmerzen nach einer Woche beträgt die Chance auf Gesundung nach drei Monaten $30 \%$. Analog, jedoch mit niedrigeren Wahrscheinlichkeitswerten, verläuft die Prognose für die erste Woche und den ersten Monat nach Erhebung der Parameter.

Fazit: Das Kreuzschmerz-Prognosemodell der Autoren erreichte in der C-Statistik einen Wert von 0,76 , der für eine gute, wenn auch nicht starke Trennschärfe steht. Angaben zu Spezifität und Sensitivität machten die Forscher nicht, vorhergesagte und beobachtete Befunde stimmten aber recht gut überein. Allerdings betonen die Autoren, das Modell müsse extern validiert werden. Zudem sei in einer randomisierten und kontrollierten Studie zu klären, ob sich mit dem Vorhersagewerkzeug die Ergebnisse für die Patienten verbessern und Kosten senken ließen.

Dr. Robert Bublak

Da Silva T et al. Predicting recovery in patients with acute low back pain: A Clinical Prediction Model. Eur J Pain 2017; doi: 10.1002/ejp.976 